\title{
Bedarfsorientierte Medizin ist keine Wunschmedizin
}

\begin{abstract}
Die Diskussion um Bedarf und Bedürfnisse im Gesundheitswesen darf nicht den Eindruck vermitteln, es werde eine wunscherfüllende Medizin angestrebt, warnte Prof. Dr. Dr. Hans Heinrich Raspe, Lübeck, in Tutzing. Sehr klar sei zu trennen zwischen dem objektivierbaren Bedarf einer medizinischen Maßnahme, also der Indikation, und den Bedürfnissen bzw. der Inanspruchnahme.
\end{abstract}

— Die Indikation müsse immer auch eine Lösung umfassen, sonst sei sie gar nicht zu stellen: „Ohne Lösung gibt es keinen technisch-medizinischen Bedarf, höchstens einen nach Begleitung oder einen Forschungsbedarf."

Die Bedürfnisse der Patienten stellen dagegen eher subjektive Wünsche dar, die nicht einmal in einer Inanspruchnahme münden müssen, wie das Beispiel chronisch entzündliche Darmerkrankungen (CED) zeigt: Die Patienten berichten häufig über hohe Stressbelastung und viele haben auch Ernährungsprobleme. Entsprechend finden die Betroffenen das Angebot von Stresstraining und Ernährungsberatung gut und wünschen sich das mehrheitlich, wie Raspe in eigenen Studien evaluiert hat. Da Stress ein wichtiger Risikofaktor für eine hohe Krankheitsaktivität ist, sind Stresstrainings auch medizinisch indiziert, und die Ernährungsberatung ist hier eine GKV-
Leistung. In Anspruch nahmen diese Angebote im Rahmen eines proaktiven Versorgungskonzepts aber nur $10 \%$ der mittelschwer und 12\% der stark durch Stress beeinträchtigten CED-Patienten. Die geringe Inanspruchnahme änderte sich auch nicht durch den ausdrücklichen Hinweis des betreuenden Arztes auf das Angebot (Abb. 1).

\section{Weniger ist mehr}

Dass eine gewisse Zurückhaltung der Ärzte nicht gleichbedeutend mit schlechter Versorgung ist, zeigt die "Less is more"-Bewegung in den USA, auf die Prof. Dr. Jürgen Windeler, Leiter des IQWiG, hinwies (http://archinte.amaassn.org/cgi/collection/less_is_more). Die medizinische Profession diskutiert hier selbst, was wesentlich und dem Patientenwohl förderlich ist oder was unnötige Kosten verursacht und dem Patienten nicht nutzt oder sogar schadet.

\section{Abbildung 1}

Beispiel: Erhöhte Stressbelastung

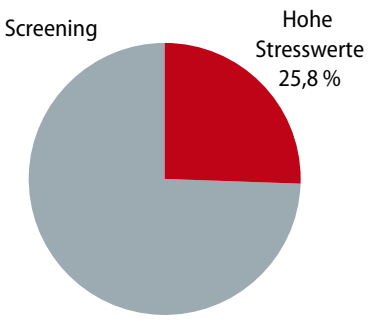

Gesamtgruppe $(\mathrm{N}=431)$

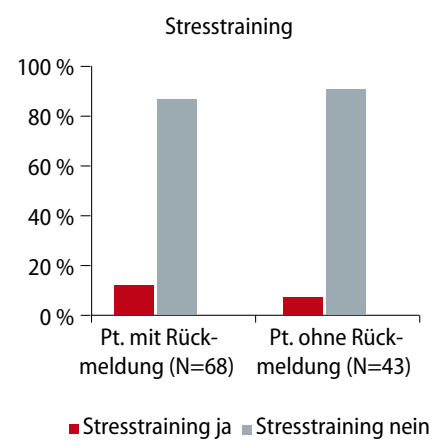

Abb. 1 Viel Stress bei CED ist Gift. Das Angebot von Stresstrainings finden Patienten deshalb gut. Wahrgenommen werden sie aber trotzdem nicht.
Auslöser dieser Diskussion war nicht primär der Kostendruck, sondern die Erkenntnis, dass immer mehr Diagnostik und Therapie auch problematisch sein kann. „Es ist ein durch die Medien weit verbreiteter Irrglauben, dass jede innovative diagnostische Maßnahme oder Medikation besser ist als das Altbewährte und Herkömmliche“, beklagte Prof. Dr. WolfDieter Ludwig aus Berlin-Buch, Vorsitzender der Arzneimittelkommission der Deutschen Ärzteschaft. „Dabei ist das Einzige, was wir wissen: Es ist gefährlicher!" Er forderte eine Rückbesinnung auf die Profession des Arztseins und verweist auf die Charta zur ärztlichen Berufsethik. Grundprinzipien des ärztlichen Handelns sind danach das Primat des Patientenwohls, das Selbstbestimmungsrecht der Patienten und die soziale Gerechtigkeit. Damit verbunden sind zehn ärztliche Verpflichtungen, nämlich die

- zur fachlichen Kompetenz

- zur Wahrhaftigkeit im Umgang mit Patienten

- zur Vertraulichkeit

- zur Pflege angemessener Beziehungen zum Patienten

- zur ständigen Qualitätsverbesserung

- zum Erhalt des Zugangs zu medizinischen Leistungen

- zur gerechten Verteilung begrenzter Mittel im Gesundheitswesen

- zur Nutzung wissenschaftlicher Erkenntnisse

- zum angemessenen Verhalten bei Interessenkonflikten

- zur kollegialen Verantwortung.

Ludwig ist sich sicher: „Wenn das zur Kenntnis genommen würde, hätten wir viele Probleme nicht!“

FrIEDERIKE KLEIN .

- Quelle: [1] Patientenforum Medizinethik, Tutzing, 28.-30. 10. 2011

[2] Köbberling J. Charta zur ärztlichen Berufsethik. Z ärztl. Fortbild Qual Sich 2003; 97: 76-9 